\title{
Progress towards the Development of a Traveling Wave Direct Energy Converter for Aneutronic Fusion Propulsion Applications
}

\author{
A. G. Tarditi \\ Electric Power Research Institute, Knoxville, TN (USA) \\ A. Chap \\ University of Maryland, College Park, MD (USA) \\ J. Wolinsky and J. H. Scott \\ NASA Johnson Space Center, M/C EP, Houston, TX (USA)
}

\begin{abstract}
A coordinated experimental and theory/simulation effort has been carried out to investigate the physics of the Traveling Wave Direct Energy Converter (TWDEC), a scheme that has been proposed in the past for the direct conversion into electricity of the kinetic energy of an ion beam generated from fusion reactions. This effort has been focused in particular on the TWDEC process in the high density beam regime, thus accounting for the ion beam expansion due to its space charge.
\end{abstract}

$\begin{array}{ll}\quad \text { Nomenclature } \\ \mathrm{kg} / \mathrm{kW} & =\text { kilograms per kilowatt-electric (specific mass) } \\ \mathrm{keV} & =\text { kilo-electron-volt (particle energy) } \\ \mathrm{A} / \mathrm{m}^{2} & =\text { amps per square meter (current density) } \\ \mathrm{AC} & =\text { alternating current } \\ D C & =\text { direct current } \\ G, T & =\text { gauss, tesla (magnetic field strength) } \\ V & =\text { volts (electric potential) }\end{array}$

\section{Introduction}

$\mathrm{T}$ HE motivation for this experimental activity, and the focus on the high density beam in particular, stems from the potential application of the TWDEC technology for MW-class aneutronic fusion space propulsion. In this case, a low power density TWDEC would require a very large structure, resulting in additional engineering constraints for a ground-based test reactor and a large mass penalty for a reactor operating on a spacecraft. Another related goal of this investigation is the understanding of the achievable conversion efficiency. Values above $70 \%$ appear necessary to enable the low specific mass $\left(<4 \mathrm{~kg} / \mathrm{kW}_{\mathrm{e}}\right)$ in-space power system required for an affordable architecture for the human exploration of Mars and beyond.

A TWDEC test article designed at NASA Johnson Space Center (JSC) has been built. It operates at beam current densities on the order of $30 \mathrm{~A} / \mathrm{m}^{2}$ and has hollow (grid-less) electrodes, a solenoidal confinement field of up to 180 $\mathrm{G}$ in a cylindrical chamber of about $0.5 \mathrm{~m}$ diameter, and a beam energy up to $1.5 \mathrm{keV}$ (a very low energy compared to that of the fusion products). However, for the purpose of this investigation the lower beam energy is not considered critical since the results can be scaled up to higher ion energy levels. A particle-in-cell (PIC) code has also been developed specifically for the simulation of the TWDEC process and has been validated with analytical comparisons.

Experimental results show the predicted capacitive coupling between a bunched beam of ions and the decelerator electrodes, generating a sustained AC potential on a resistive load. Quantitative comparisons of the ion bunch 
dynamics simulations with the PIC simulation are presented. A preliminary investigation on the effect of a partially neutralizing electron beam, generated near the ion source and confined along the beam axis, is also discussed.

\section{The Experimental Setup}

The TWDEC concept, as originally formulated [Momota, 1992], is based on the interaction of a charged particle bunch that travels through a conducting structure. Gridded electrodes were used in early experiments (e.g. [Takeno, 2000], [Sugihara, 2006]). As the ion bunches travel near the conductors, they induce charge fluctuations that translate into an alternating electric potential between two adjacent electrodes.

A conceptual scheme of the TWDEC concept is shown in Figure 1.

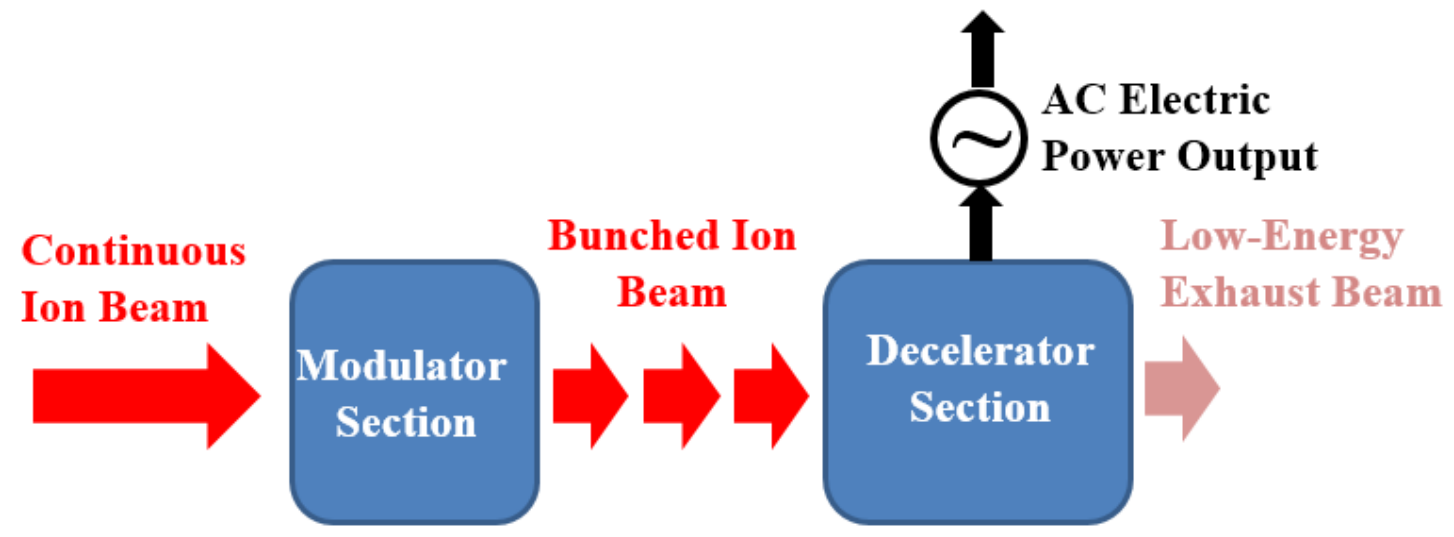

Figure 1 - TWDEC conceptual scheme.

In this investigation, due to the focus on high density beams, the electrodes consist of rings with the inner radius slightly larger than the estimated beam cross section (represented as a yellow shade in Figure 3-a). Figure 3-b shows the actual installation in the vacuum chamber of four aluminum rings with a $10 \mathrm{~cm}$ inner diameter.

The experimental setup for the study of the TWDEC process that has been assembled at the NASA Johnson Space Center has been previously described in [Chap, 2014]. Recently, the test article has been upgraded with the introduction of a larger magnetic coil capable of producing a magnetic field on axis of about $0.02 \mathrm{~T}$ (Figure 2). 


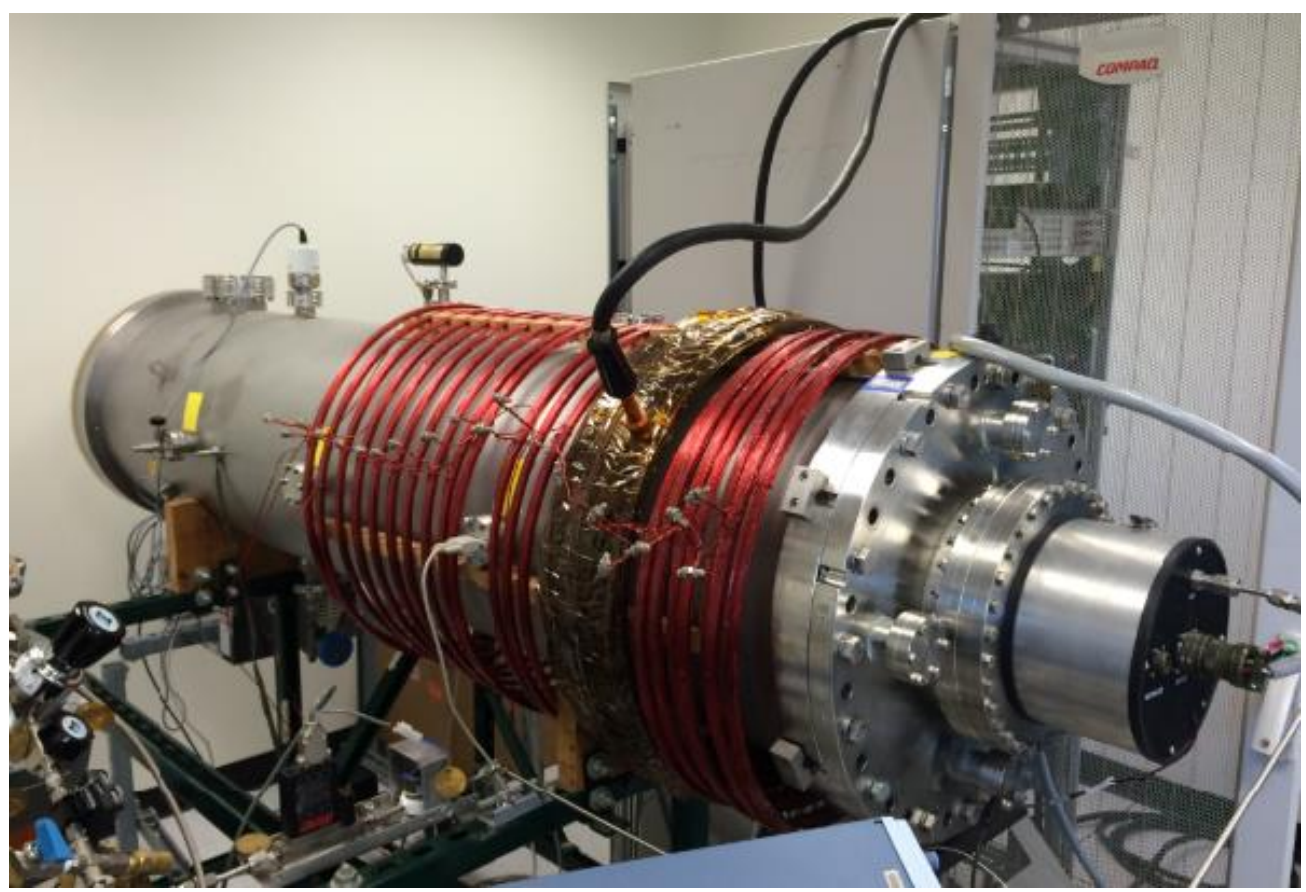

Figure 2 - NASA-JSC TWDEC physics experiment.

The introduction of this magnetic field coil allowed a significant improvement in the performance of the energy conversion process by enabling increased beam collimation.

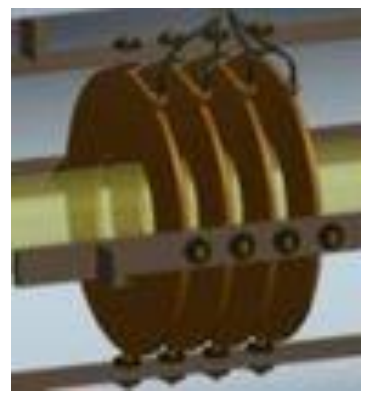

a)

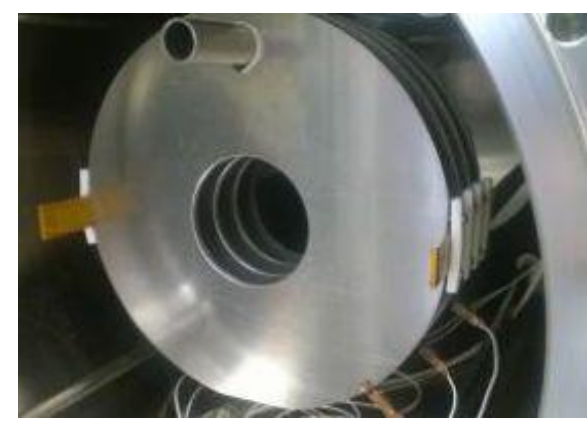

b)

Figure 3 - Conceptual design and implementation of ring shaped electrodes scheme.

For the tests herein reported, the beam modulation and deceleration stages are adjacent to each other and consist of the stack of four electrodes, as shown in Figure 3. 
In this configuration, the first two electrodes are utilized for the beam modulation, while the third and fourth electrodes are used for the deceleration. For the modulation of the beam into bunches, electrode \#2 is switched from +400 to $0 \mathrm{~V}$ with respect to electrode \#1. All electrodes are floating with respect to ground.

The electrical schematic of the experiment is shown in Figure 4. The switches $S_{1}$ and $S_{2}$ are fast MOSFET transistors controlled by a variable duty cycle square pulse generator. When $S_{1}$ is closed, $S_{2}$ is open and the floating power supply DC voltage $\mathrm{V}_{\text {mod }}$ is applied to the modulator electrodes, an electric field is created that repels the ions. Then $S_{1}$ is opened and $S_{2}$ is closed, thus discharging the capacitance between the two electrodes and allowing for the beam to transit.

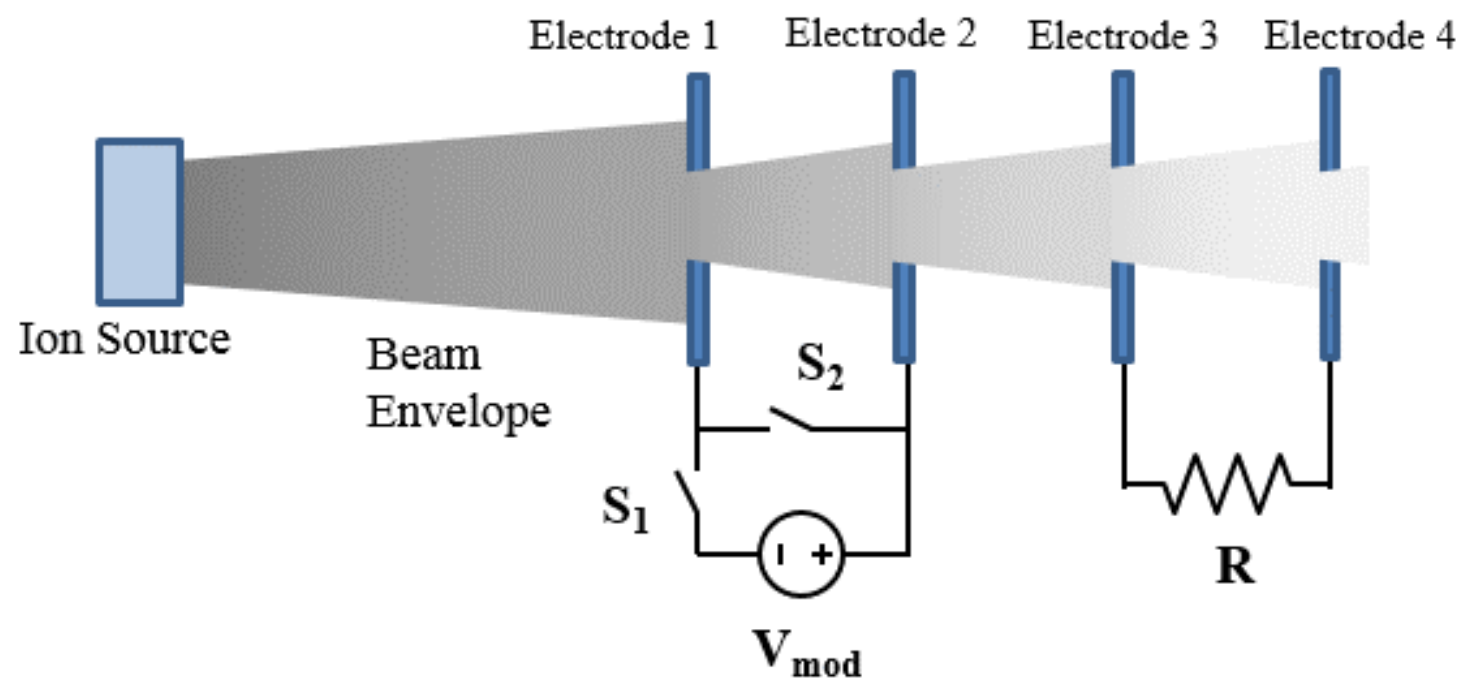

Figure 4 - TWDEC four-electrode connections. The beam envelope expansion is not computed but shown for illustration purposes to enhance the density variations.

\section{The Beam Source}

The ion source produces a beam of Helium ions, simulating the product of certain nuclear fusion reactions. The collimation of the ion beam as it leaves the source is improved by the presence of an electron neutralizer. This neutralizer consists of a hot filament placed slightly past the outer grid of the ion source. The neutralizer configuration has been modified with respect to the original manufacturer's design as shown in Figure 5 (the filament is set in a coiled circular shape near the axis of the source). 

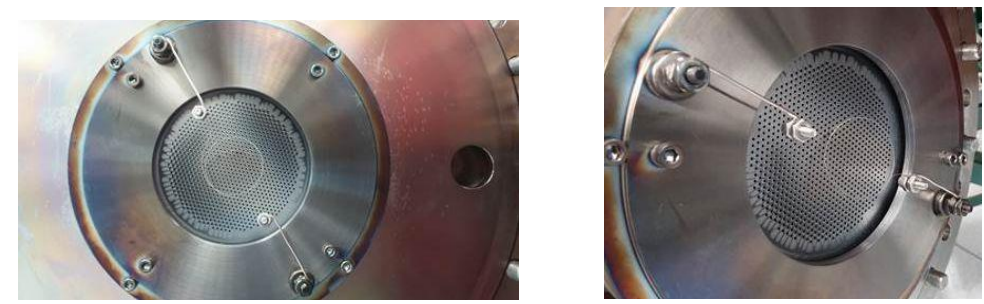

Figure 5 - Front and side-angle view of the electron neutralizer filament placed near the outer grid of the ion source.

The electrons are then mostly generated in the inner part of the beam, while the ions are spread more uniformly through the entire beam cross section. This arrangement is meant to favor the generation of a beam characterized by a non-neutralized radial charge density profile, with (positive) ion excess towards the outer diameter.

The presence of the magnetic field helps to maintain beam collimation further downstream (in the electrode region), as the electrons ("boiled off" at low energy from the heated filament) will be confined in the central region with a very small gyro radius.

A Langmuir probe has been designed for the purpose of determining the impact of the electron neutralizer on the beam charge density profile, and measurements are planned for future experiments. The probe can be accurately positioned through a motorized linear actuator. So far, only preliminary probe tests have been performed, with the probe placed in an axial position downstream of the decelerator section (far from the ion source).

\section{Experimental Results}

The modulator MOSFET transistors are limited to peak voltages up to $600 \mathrm{~V}$. In order to allow margin, the modulator has to date been operated only up to $400 \mathrm{~V}$. By regulating the ion source for a $400 \mathrm{eV}$ energy, theoretically up to a $100 \%$ beam modulation can be achieved.

The magnetic field coil is supplied with a 200 A continuous current. Based on the coil design specifications, this current corresponds to a peak axis magnetic field of about 180 Gauss. The length of the coil covers the stack of all four electrodes, enhancing the beam collimation throughout the entire modulation/deceleration process. The effect of the magnetic field has been verified by comparing a Langmuir probe-type measurement on the beam downstream the decelerator section with and without the magnetic field.

In order to study the energy conversion process, the voltage on the resistor placed between electrodes 3 and 4 is measured while electrodes 1 and 2 are used for the modulation.

The modulator driver is set to operate the switches to produce a "beam on/beam off" duty cycle ratio of less than 1 to 50 . In this condition, the ion beam bunches are generated with a less than $1 \mu$ s beam-on switching.

As a reference, the time required for the ion bunch to cross the distance between two electrodes placed at distance $\Delta \mathrm{x}=5 \mathrm{~cm}$ is $\Delta \mathrm{t}=0.36 \mu \mathrm{s}^{1}$, thus the bunch axial length is longer than the electrode spacing. In future experiments, the goal is to test with a shorter axial bunch length. However, this is not critical to the results presented in this paper.

${ }^{1} \Delta \mathrm{t}=\Delta \mathrm{x} / \mathrm{v}_{\mathrm{i}}, \mathrm{v}_{\mathrm{i}}=\left(2 \mathrm{~W}_{\mathrm{i}} / \mathrm{m}_{\mathrm{i}}\right)^{1 / 2}$ where $\mathrm{W}_{\mathrm{i}}, \mathrm{m}_{\mathrm{i}}$ and $\mathrm{v}_{\mathrm{i}}$ are the beam ion energy, mass and speed respectively. By considering the single-charged Helium ions: $\mathrm{m}_{\mathrm{i}}=4 \cdot 1.67 \cdot 10^{-27} \mathrm{Kg}=6.68 \cdot 10^{-27} \mathrm{~kg}, \mathrm{~W}_{\text {beam }}=400 \mathrm{eV} \cdot 1.6 \cdot 10^{-19} \mathrm{~J} / \mathrm{eV}=$ $6.4 \cdot 10^{-17} \mathrm{~J}$, then $\mathrm{v}_{\text {beam }}=1.38 \cdot 10^{5} \mathrm{~m} / \mathrm{s}$ and $\mathrm{t}_{\text {cross }}=0.05 /\left(2.19 \cdot 10^{5}\right)=2.32 \cdot 10^{-7} \mathrm{~s}=0.36 \mu \mathrm{s}$. 
It is important to understand the entire extent of the interaction of the beam with the electrodes to evaluate the actual efficiency of the TWDEC process. If the beam is not perfectly confined as it passes through the holes, the electrodes will be impacted by some particles and collect charges.

As sketched in Figure 4, due to the beam expansion, each electrode will be affected by a progressively lower density beam impinging on its surface. Thus, for example, the fourth electrode will collect less charge than the third, leading to a potential difference between the electrodes even without the presence of the modulation (i.e., without the presence of the "TWDEC effect"). This results in a loss in conversion efficiency, but, according to TWDEC theory, it can be reduced by increasing the magnetic field (which provides electron confinement and reduces the beam density variation along the axis).

The signal measured on the resistor in the decelerator section from the latest experimental runs is shown in Figure 6. As a baseline reference, the same signal is shown in Figure 7 without the beam, showing the noise induced by capacitive coupling among the electrodes.

The signal in Figures 6 and 7 is collected through a 50x probe, so the $10 \mathrm{mV} / \mathrm{div}$ on the scope screen corresponds to $500 \mathrm{mV} / \mathrm{div}$, and the waveform shows about $2 \mathrm{~V}$ peak-to-peak. The resistor was set to $100 \Omega$ to lower the capacitively coupled noise, and $40 \mathrm{~mW}$ of power is thus shown to be converted from the beam bunch. Note that the present experiment is by no means optimized for power conversion efficiency.

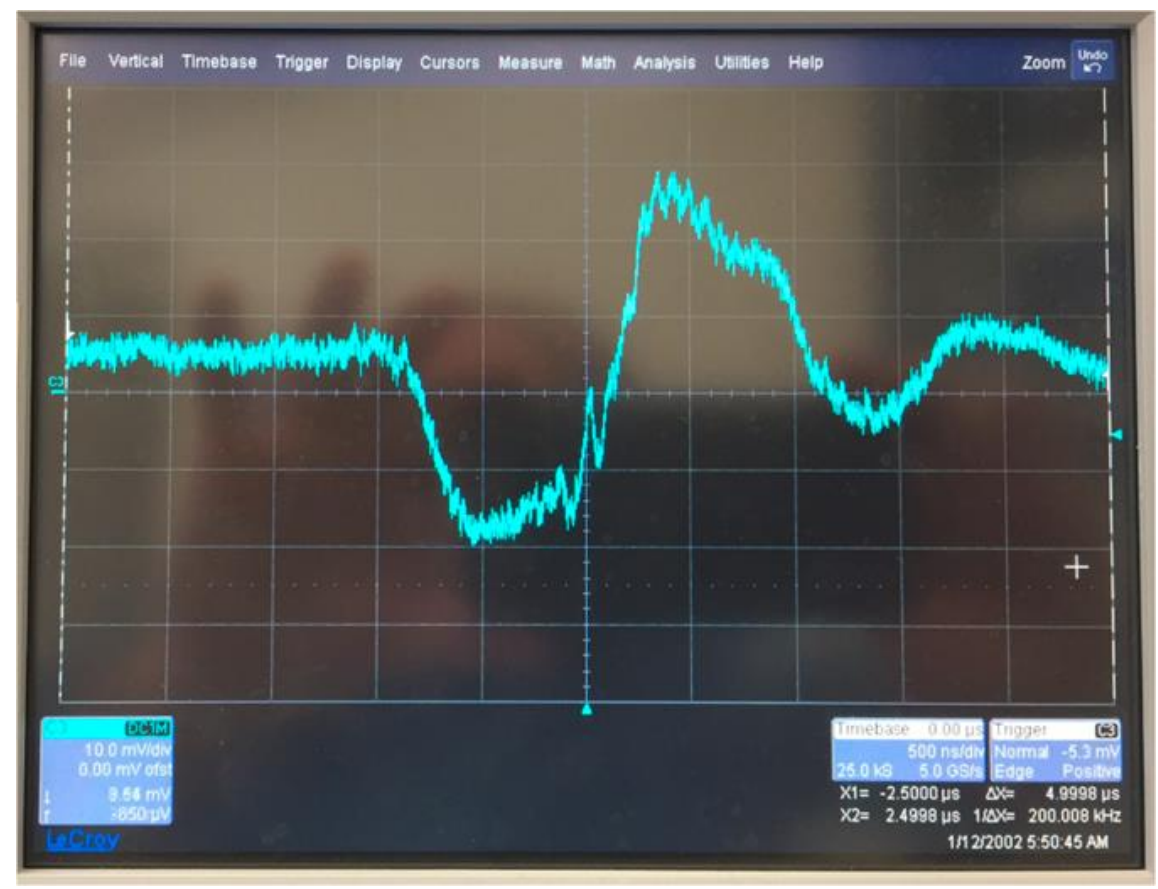

Figure 6 - Waveform collected on the resistor placed in between the decelerator electrodes. This case had a $180 \mathrm{Gs}$ magnetic field, a beam current of $35 \mathrm{~mA}$, and a beam energy of $400 \mathrm{eV}$ with $100 \%$ modulation. 


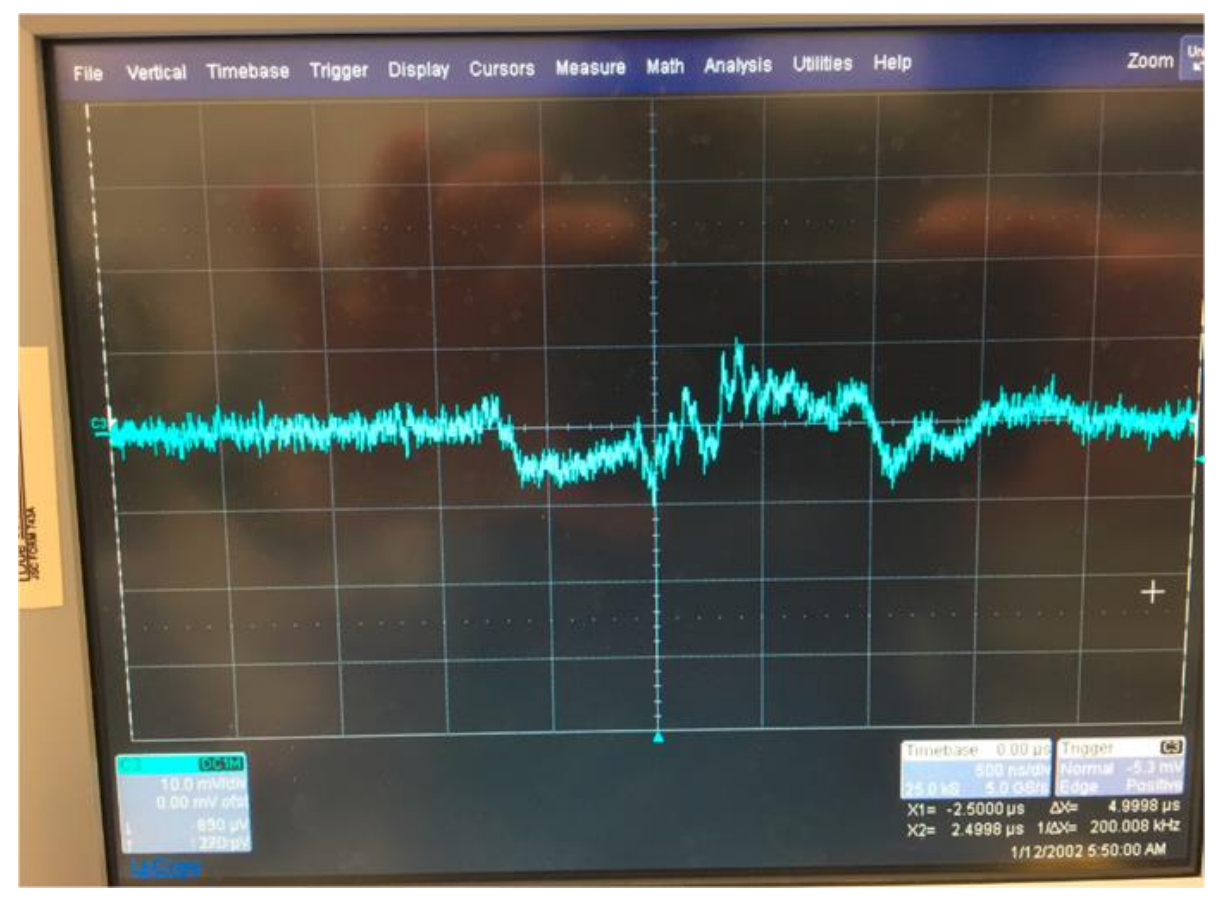

Figure 7 - Waveform collected on the decelerator resistor without the beam, to show the baseline noise capacitively induced by the modulator signal.

\section{Simulation Studies}

A PIC code has been developed [Chap, 2013], [Chap, 2014] for the purpose of analyzing the beam dynamics in the TWDEC and eventually performing optimization studies. An important element for the analysis of the energy conversion process is the characterization of the beam profile.

PIC code simulations of the ion beam expansion along the axial dimension have been performed for validation purposes, and compared with theoretical predictions.

The ion beam expansion can be described according to the paraxial equation model [Humpries, 1990] for the expansion of a beam due to space charge:

$\frac{d^{2} R}{d z^{2}}=\left[\frac{e I}{2 \pi \epsilon_{0} m v^{\mathrm{a}}}\right] \frac{1}{R}$

Where $\mathrm{R}$ is beam radius, $\mathrm{z}$ the axial dimension, $\mathrm{I}$ the beam current, $\mathrm{v}$ the beam velocity, e the particle charge and $\mathrm{m}$ the particle mass.

Predictions from Eq. 5.1 compare well with the PIC model for the same beam conditions, as shown in Fig. 8. 


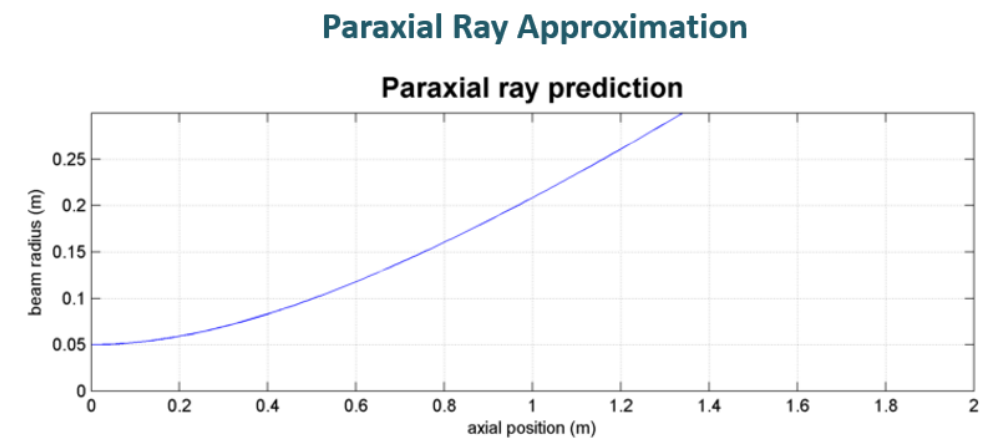

Simulation

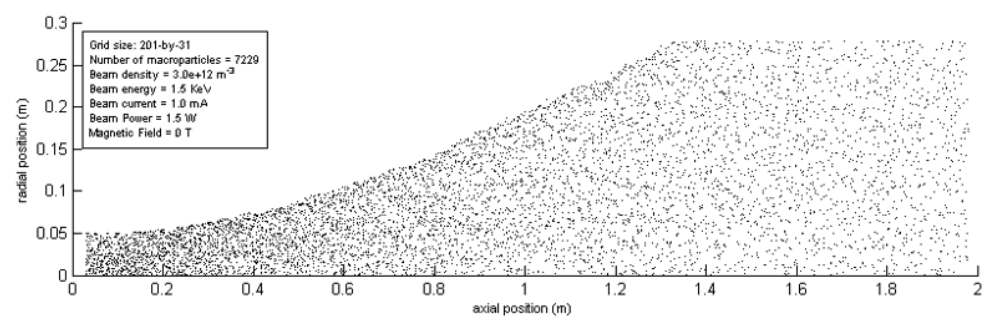

Figure 8 - A comparison between the simulation and the theoretical model of an ion beam expansion due to its own space charge.

The paraxial ray model can also be used in the presence of a confining magnetic field,

$\frac{d^{2} R}{d z^{2}}=\left[\frac{e I}{2 \pi \epsilon_{0} m v^{3}}\right] \frac{1}{R}+\left[\frac{q B_{z}}{2 m v}\right]^{2}\left(\frac{\left(R_{0}\right)^{4}}{R^{3}}-R\right)$

and comparison with the PIC simulation is shown in Figure 9, showing an excellent agreement. 


\section{Theory: paraxial ray equation, $0.4 \mathrm{~T}$ magnetic field}

\section{$1500 \mathrm{eV}, 20 \mathrm{~mA}$}
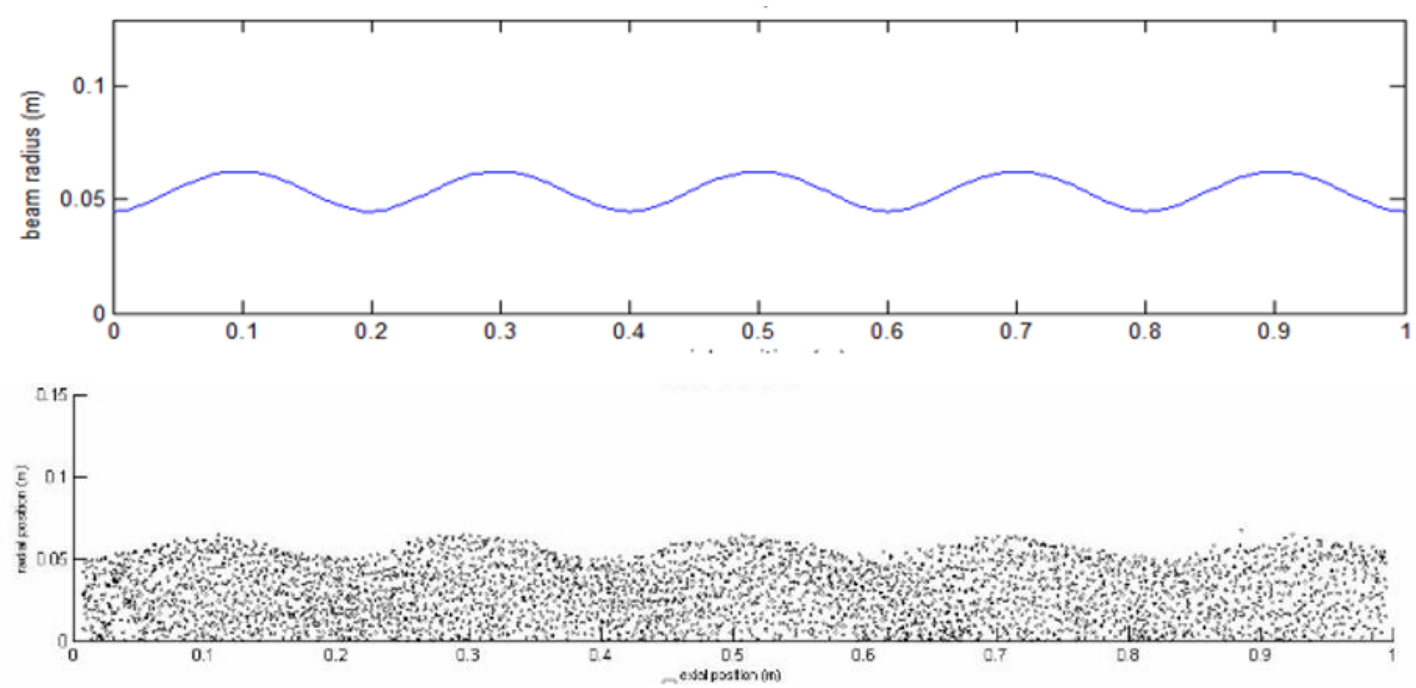

Figure 9 - A comparison between the simulation and the theoretical model of an ion beam expansion due to its own space charge in the presence of a magnetic field.

\section{Acknowledgments}

The authors gratefully acknowledge the National Aeronautics and Space Adminstration for its support to this research.

\section{Conclusions}

The investigation of the TWDEC process in the high-density ion beam regime is still in progress, but it has provided very encouraging results so far. With a modest investment for additional beam/plasma diagnostics, important issues like the conversion efficiency vs. beam density and the impact of the neutralizing electrons can be addressed.

The present work may lead to the development of a basic enabler technology for the development of aneutronic fusion power reactors.

\section{References}

[Chap, et al. 2013] A. M. Chap et al., “A Particle-in-cell Simulation for the Traveling Wave Direct Energy Converter (TWDEC) for Fusion Propulsion" (AIAA 2013-3912) 49th AIAA/ASME/SAE/ASEE Joint Propulsion Conference, 2013, $10.2514 / 6.2013-39$

[Chap, et al. 2014] AIAA 2014-3559

[Momota, 1992] Fusion Technology, 21, 2307 (1992)

[Takeno, 2000] H. Takeno, Y. Ikeda, T. Yamada, K. Noda and Y. Yasaka, Jpn. J. Appl. Phys. 39, 5287 (2000).

[S. Humphries, 1999] Principles of Charged Particle Acceleration, Wiley (1999)

[Sugihara, 2006] K. Sugihara, et al., "Deceleration Experiment on a Simulator of Traveling Wave Direct Energy Converter for D3He Fusion Reactor" Journal of the Korean Physical Society, Vol. 49, December 2006, pp. S141-S145 (2006) 